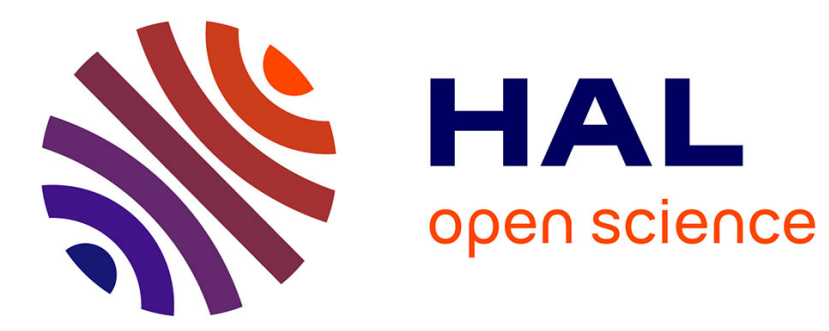

\title{
Exponential functionals of Brownian motion and explosion times of a system of semilinear SPDEs
}

\author{
M Dozzi, E. T. Kolkovska, José Alfredo Lopez
}

\section{To cite this version:}

M Dozzi, E. T. Kolkovska, José Alfredo Lopez. Exponential functionals of Brownian motion and explosion times of a system of semilinear SPDEs. Stochastic Analysis and Applications, 2013, 31 (6), pp.975-991. 10.1080/07362994.2013.817232 . hal-01095641

\section{HAL Id: hal-01095641 \\ https://hal.science/hal-01095641}

Submitted on 16 Dec 2014

HAL is a multi-disciplinary open access archive for the deposit and dissemination of scientific research documents, whether they are published or not. The documents may come from teaching and research institutions in France or abroad, or from public or private research centers.
L'archive ouverte pluridisciplinaire HAL, est destinée au dépôt et à la diffusion de documents scientifiques de niveau recherche, publiés ou non, émanant des établissements d'enseignement et de recherche français ou étrangers, des laboratoires publics ou privés. 


\title{
Exponential Functionals of Brownian Motion and Explosion Times of a System of Semilinear SPDEs
}

\author{
M. Dozzi* $\quad$ E.T. Kolkovska ${ }^{\dagger} \quad$ J.A. López-Mimbela ${ }^{\dagger}$
}

\begin{abstract}
We investigate lower and upper bounds for the blowup times of a system of semilinear SPDEs. Under certain conditions on the system parameters, we obtain explicit solutions of a related system of random PDEs, which allows us to use a formula due to Yor to obtain the distribution functions of several explosion times. We also give the Laplace transforms at independent exponential times of related exponential functionals of Brownian motion.
\end{abstract}

2000 Mathematics Subject Classifications: 35R60, 60H15, 74 H35

Key words and phrases: Blowup of systems of semi-linear equations, systems of stochastic partial differential equations, weak and mild solutions

\section{Introduction}

Existence and nonexistence of global solutions for semilinear parabolic equations was investigated initially by Fujita [4], who proved that for a bounded smooth domain $D \subset \mathbb{R}^{d}$, the equation

$$
\frac{\partial u(t, x)}{\partial t}=\Delta u(t, x)+u^{1+\beta}(t, x), \quad x \in D,
$$

with Dirichlet boundary condition, where $\beta>0$ is a constant, explodes in finite time for all nonnegative initial values $u(0, x) \in L^{2}(D)$ satisfying $\int_{D} u(0, x) \psi(x) d x>\Lambda^{1 / \beta}$. Here $\Lambda>0$ is the first eigenvalue of the Laplacian on $D$ and $\psi$ the corresponding eigenfunction normalized so that $\|\psi\|_{L^{1}}=1$.

In a previous work [2] we investigated blow-up times of semilinear SPDEs of the prototype

$$
d u(t, x)=\left(\Delta u(t, x)+u^{1+\beta}(t, x)\right) d t+\kappa u(t, x) d W_{t}, \quad x \in D,
$$

with Dirichlet boundary conditions, where $\left\{W_{t}\right\}$ is a standard one-dimensional Brownian motion. We obtained bounds both for the probability of finite-time blow-up of $u(t, x)$, and for the probability of nonexplosion of $u(t, x)$ in finite time. In case of $\kappa=0$, the bounds we found give the result of Fujita quoted above. We refer to [2] for definitions of blow-up times, and for types of solutions of SPDEs. In [2] it is also shown that the asymptotic behavior of (1.1) is determined to a great extent by the distribution of the exponential functional

$$
\int_{0}^{t} \exp \left\{\kappa \beta W_{r}-\beta\left(\Lambda+\kappa^{2} / 2\right) r\right\} d r
$$

\footnotetext{
*Institut Elie Cartan, Université de Lorraine, B.P. 239, F-54506 Vandoeuvre-lès-Nancy Cedex, France.

${ }^{\dagger}$ Centro de Investigación en Matemáticas, Apartado Postal 402, 36000 Guanajuato, Mexico.
} 
Functionals of the above form arise in many applications, specially in Financial and Actuarial Mathematics $[3,6,14]$ and have been investigated by different methods and by various authors $[1,3,7,8,9,12,13,14]$.

From the results of [2] it follows that for initial values of the form $u(0, x)=k \psi(x), x \in D$, where $k>0$ is a parameter, the explosion time $\varrho$ of (1.1) satisfies $\sigma_{*} \leq \varrho \leq \sigma^{*}$, where

$$
\begin{aligned}
\sigma_{*} & =\inf \left\{t \geq 0: \int_{0}^{t} \exp \left\{\kappa \beta W_{r}-\beta\left(\Lambda+\kappa^{2} / 2\right) r\right\} d r \geq 1 /\left(\beta k^{\beta}\|\psi\|_{\infty}^{\beta}\right)\right\} \\
\sigma^{*} & =\inf \left\{t \geq 0: \int_{0}^{t} \exp \left\{\kappa \beta W_{r}-\beta\left(\Lambda+\kappa^{2} / 2\right) r\right\} d r \geq 1 /\left(\beta k^{\beta}\left(\int \psi^{2}(x) d x\right)^{\beta}\right)\right\} .
\end{aligned}
$$

Let us mention that the density of $\int_{0}^{t} \exp \left\{\kappa \beta W_{r}-\beta\left(\Lambda+\kappa^{2} / 2\right) r\right\} d r, t>0$, can be obtained from Yor's formula ([14], Ch. 2 and [13], Proposition 2; see also [8]). The upper bound $\sigma^{*}$ of $\varrho$ was achieved in [2] by determining the blow-up time of a subsolution of Eq. (1.1), while for the lower bound $\sigma_{*}$, a scheme of successive approximations for the mild solution of (1.1) was used.

Our aim in this paper is to obtain lower and upper bounds for the blow-up times of the system of semilinear SPDEs

$$
\begin{aligned}
& d u_{1}(t, x)=\left[\left(\Delta+V_{1}\right) u_{1}(t, x)+u_{2}^{p}(t, x)\right] d t+\kappa_{1} u_{1}(t, x) d W_{t} \\
& d u_{2}(t, x)=\left[\left(\Delta+V_{2}\right) u_{2}(t, x)+u_{1}^{q}(t, x)\right] d t+\kappa_{2} u_{2}(t, x) d W_{t}, \quad x \in D,
\end{aligned}
$$

with Dirichlet boundary conditions

$$
u_{i}(0, x)=f_{i}(x) \geq 0, x \in D \text { and } u_{i}(t, x)=0, t \geq 0, x \in \partial D, \quad i=1,2 .
$$

Here $p \geq q>1$ are constants, $D \subset \mathbb{R}^{d}$ is a bounded smooth domain, $V_{i}>0$ and $\kappa_{i} \neq 0$ are constants, $i=1,2$, and $\left\{W_{t}\right\}$, is a standard one-dimensional Brownian motion defined on some probability space $(\Omega, \mathcal{F}, \mathbb{P})$. As in $[2]$, we are going to consider weak solutions of (1.2)-(1.3).

Below we are going to take the initial values of the form $f_{i}=k_{i} \psi$ for some constants $k_{i}>0$, $i=1,2$. Also, we are going to set $V_{i}=\Lambda+\kappa_{i}^{2} / 2, i=1,2$. These choices make it possible to obtain explicit solutions of a related system of random PDEs, and to use Yor's formula and some extensions of it. Moreover, in contrast with the case treated in [2], the blow-up times of (1.2) are finite with probability one; see e.g. [7], Prop. 6.4 and [9], Section 2. For general constants $\kappa_{1}, \kappa_{2}$ and $p \geq q>1$, we obtain random times $\tau_{* *}, \tau^{* *}$ such that $\tau_{* *} \leq \tau \leq \tau^{* *}$, where $\tau$ is the blow-up time of (1.2). Moreover, we show that the distributions of $\tau_{* *}$ and $\tau^{* *}$ are given in terms of exponential functionals of the form

$$
\mathcal{A}^{* *}(t)=\int_{0}^{t} e^{a W_{r}} \wedge e^{b W_{r}} d r \quad \text { and } \quad \mathcal{A}_{* *}(t)=\int_{0}^{t} e^{a W_{r}} \vee e^{b W_{r}} d r .
$$

By adapting a method of Jeanblanc, Pitman and Yor [5] to our setting, we obtain the Laplace transforms of $\mathcal{A}^{* *}\left(\theta_{\lambda}\right)$ and $\mathcal{A}_{* *}\left(\theta_{\lambda}\right)$ at random times $\theta_{\lambda}$ which are exponentially distributed with parameter $\lambda$, for any value of $\lambda>0$. From this we can derive upper bounds for probabilities of the form $\mathbb{P}\left\{\tau \geq \theta_{\lambda}\right\}$ for each $\lambda>0$.

The paper is organized as follows. In Section 2, we use a random transformation of System (1.2) to obtain a related system of random partial differential equations which is useful to obtain 
lower and upper bounds for $\tau$. The special case when $p \kappa_{2}-\kappa_{1}=q \kappa_{1}-\kappa_{2}=: \rho$ is dealt with in Section 3; see theorems 1 and 2 below. In this special setting the relevant exponential functional is of the form $\int_{0}^{t} e^{\rho W_{r}} d r$, and the distributions of $\tau_{* *}$ and $\tau^{* *}$ can be obtained explicitly from Yor's formula. The semi-linear system without the condition $p \kappa_{2}-\kappa_{1}=q \kappa_{1}-\kappa_{2}$ is studied in sections 4 and 5; see theorems 3 and 5, and Corollary 4. We point out that Theorem 1 is in fact a special case of Theorem 3 and its Corollary 4, and Theorem 2 is a special case of Theorem 5 . The reason why we treat the case of Section 3 separately is that the key points of the proofs of theorems 3 and 5 appear more clearly and more simply in the proofs of theorems 1 and 2 .

For a system of the form (1.2) with $V_{1}=V_{2}=0$, similar results can be obtained for $\tau^{* *}$. However, in this case $\tau_{* *}$ is given in terms of the solution of a non-homogeneous random Bernoulli equation for which we were not able to find an explicit solution.

\section{A system of random PDEs}

Let us define for $i=1,2$,

$$
v_{i}(t, x)=\exp \left\{-\kappa_{i} W_{t}\right\} u_{i}(t, x), \quad t \geq 0, x \in D .
$$

By Ito's formula,

$$
e^{-\kappa_{i} W_{t}}=1-\kappa_{i} \int_{0}^{t} e^{-\kappa_{i} W_{s}} d W_{s}+\frac{\kappa_{i}^{2}}{2} \int_{0}^{t} e^{-\kappa_{i} W_{s}} d s
$$

Putting $u_{i}\left(t, \varphi_{i}\right)=\int_{D} u_{i}(t, x) \varphi_{i}(x) d x, i=1,2$, where $\varphi_{i}$ is any smooth function with compact support, we get that a weak solution of (1.2) is given by

$$
u_{i}\left(t, \varphi_{i}\right)=u_{i}\left(0, \varphi_{i}\right)+\int_{0}^{t} u_{i}\left(s,\left(\Delta+V_{i}\right) \varphi_{i}\right) d s+\int_{0}^{t} u_{j}^{\beta_{i}}\left(s, \varphi_{i}\right) d s+\kappa_{i} \int_{0}^{t} u_{i}\left(s, \varphi_{i}\right) d W_{s},
$$

where $\{j\}=\{1,2\} \backslash\{i\}$ and $\beta_{1}=p, \beta_{2}=q$. By applying the integration by parts formula we get that

$$
\begin{aligned}
v_{i}\left(t, \varphi_{i}\right) & :=\int_{D} v_{i}(t, x) \varphi_{i}(x) d x \\
& =v_{i}\left(0, \varphi_{i}\right)+\int_{0}^{t} e^{-\kappa_{i} W_{s}} d u_{i}\left(s, \varphi_{i}\right) \\
& +\int_{0}^{t} u_{i}\left(s, \varphi_{i}\right)\left(-\kappa_{i} e^{-\kappa_{i} W_{s}} d W_{s}+\frac{\kappa_{i}^{2}}{2} e^{-\kappa_{i} W_{s}} d s\right)+\left[e^{-\kappa_{i} W_{\cdot}}, u_{i}\left(\cdot, \varphi_{i}\right)\right](t),
\end{aligned}
$$

where the quadratic variation is given by $\left[e^{-\kappa_{i} W}, u_{i}\left(\cdot, \varphi_{i}\right)\right](t)=-\kappa_{i}^{2} \int_{0}^{t} e^{-\kappa_{i} W_{s}} u_{i}\left(s, \varphi_{i}\right) d s, t \geq 0$. Therefore,

$$
\begin{aligned}
v_{i}\left(t, \varphi_{i}\right)= & v_{i}\left(0, \varphi_{i}\right)+\int_{0}^{t} v_{i}\left(s,\left(\Delta+V_{i}\right) \varphi_{i}\right) d s \\
& +\int_{0}^{t} e^{-\kappa_{i} W_{s}}\left(e^{\kappa_{j} W_{s}} v_{j}\right)^{\beta_{i}}\left(s, \varphi_{i}\right) d s-\frac{\kappa_{i}^{2}}{2} \int_{0}^{t} e^{-\kappa_{i} W_{s}} u_{i}\left(s, \varphi_{i}\right) d s \\
= & v_{i}\left(0, \varphi_{i}\right)+\int_{0}^{t}\left[v_{i}\left(s,\left(\Delta+V_{i}\right) \varphi_{i}\right)-\frac{\kappa_{i}^{2}}{2} v_{i}\left(s, \varphi_{i}\right)\right] d s \\
& +\int_{0}^{t} e^{-\kappa_{i} W_{s}}\left(e^{\kappa_{j} W_{s}} v_{j}\right)^{\beta_{i}}\left(s, \varphi_{i}\right) d s
\end{aligned}
$$


Hence, the vector $\left(v_{1}(t, x), v_{2}(t, x)\right)$ is a weak solution of the system

$$
\frac{\partial v_{i}(t, x)}{\partial t}=\left(\Delta+V_{i}-\kappa_{i}^{2} / 2\right) v_{i}(t, x)+e^{-\kappa_{i} W_{t}}\left(e^{\kappa_{j} W_{t}} v_{j}(t, x)\right)^{\beta_{i}}, \quad v_{i}(0, x)=f_{i}(x), i=1,2,
$$

whose integral form is

$$
v_{i}(t, x)=e^{t\left(V_{i}-\kappa_{i}^{2} / 2\right)} T_{t} f_{i}(x)+\int_{0}^{t} e^{r\left(V_{i}-\kappa_{i}^{2} / 2\right)} T_{r}\left[e^{-\kappa_{i} W_{t-r}}\left(e^{\kappa_{j} W_{t-r}} v_{j}(t-r, \cdot)\right)^{\beta_{i}}\right](x) d r
$$

for $t \geq 0$, and $i=1,2$. Here $\left\{T_{t}\right\}$ is the semigroup of bounded linear operators given by

$$
T_{t} f(x)=\mathbb{E}\left[f\left(X_{t}\right), t<\tau_{D} \mid X_{0}=x\right], x \in D,
$$

for all bounded and measurable $f: D \rightarrow \mathbb{R}$, where $\left\{X_{t}\right\}_{t \geq 0}$ is the $d$-dimensional Brownian motion with variance parameter 2 , killed at the time $\tau_{D}$ at which it hits $\partial D$. As above, we denote by $\Lambda>0$ the first eigenvalue of the Laplacian on $D$, which satisfies

$$
\Delta \psi(x)=-\Lambda \psi(x), x \in D
$$

$\psi$ being the corresponding eigenfunction, which is strictly positive on $D$ and $\left.\psi\right|_{\partial D}=0$. Recall that $T_{t} \psi=e^{-\Lambda t} \psi, t \geq 0$. We are going to assume that $\psi$ is normalized so that $\int_{D} \psi(x) d x=1$.

We write $\tau$ for the blow up time of system (2.4) when the initial values are of the form $f_{1}=L(1) \psi$ and $f_{2}=L(2) \psi$ for some positive constants $L(1)$ and $L(2)$. Due to $(2.1)$ and to the a.s. continuity of Brownian paths, $\tau$ is also the explosion time of system (1.2) with initial values of the above form.

\section{$3 \quad$ The case $p \kappa_{2}-\kappa_{1}=q \kappa_{1}-\kappa_{2}$}

First we consider Eq. (1.2) with parameters

$$
p \geq q>1, \quad V_{i}=\Lambda+\frac{\kappa_{i}^{2}}{2}, i=1,2, \quad \text { and } \quad p \kappa_{2}-\kappa_{1}=q \kappa_{1}-\kappa_{2}=: \rho .
$$

We are going to find random times $\tau_{*}$ and $\tau^{*}$ which are given in terms of the exponential functional $\mathcal{A}_{t}:=\int_{0}^{t} e^{\rho W_{s}} d s, t \geq 0$, and such that $0 \leq \tau_{*} \leq \tau \leq \tau^{*}$. The density function of $\mathcal{A}_{t}$ is given explicitly in [14], Section 4.

\subsection{A lower bound for $\tau$}

We are going to obtain a random time $\tau_{*}$ which satisfies $\tau_{*} \leq \tau$.

Theorem 1 Assume conditions (3.1), and let the initial values be of the form

$$
f_{1}=L(1) \psi \text { and } f_{2}=L(2) \psi
$$

for some positive constants $L(1)$ and $L(2)$. Let $\tau_{*}$ be given by

$$
\tau_{*}=\inf \left\{t \geq 0: \int_{0}^{t} \exp \left\{\rho W_{r}\right\} d r \geq \min \left\{\frac{1}{(p-1) L^{p-1}(1)\|\psi\|_{\infty}^{p-1}}, \frac{1}{(q-1) L^{q-1}(2)\|\psi\|_{\infty}^{q-1}}\right\}\right\} .
$$

Then $\tau_{*} \leq \tau$. 
Proof. Let $v_{1}$ and $v_{2}$ solve (2.4). Then we have

$$
\begin{aligned}
& v_{1}(t, x)=e^{\Lambda t} T_{t} f_{1}(x)+\int_{0}^{t} e^{\Lambda(t-r)} T_{t-r}\left[e^{\rho W_{r}} v_{2}^{p}(r, x)\right] d r \\
& v_{2}(t, x)=e^{\Lambda t} T_{t} f_{2}(x)+\int_{0}^{t} e^{\Lambda(t-r)} T_{t-r}\left[e^{\rho W_{r}} v_{1}^{q}(r, x)\right] d r, \quad x \in D, \quad t \geq 0 .
\end{aligned}
$$

We define the operators $\mathcal{R}_{1}, \mathcal{R}_{2}$ by

$$
\begin{aligned}
& \mathcal{R}_{1} v(t, x)=e^{\Lambda t} T_{t} f_{1}(x)+\int_{0}^{t} e^{\rho W_{r}} e^{\Lambda(t-r)}\left(T_{t-r} v\right)^{p} d r \\
& \mathcal{R}_{2} v(t, x)=e^{\Lambda t} T_{t} f_{2}(x)+\int_{0}^{t} e^{\rho W_{r}} e^{\Lambda(t-r)}\left(T_{t-r} v\right)^{q} d r, \quad x \in D, \quad t \geq 0,
\end{aligned}
$$

where $v$ is any nonnegative, bounded and measurable function. Moreover, on the set $t \leq \tau_{*}$ we put

$$
\begin{aligned}
& \mathcal{B}_{1}(t)=\left[1-(p-1) \int_{0}^{t} e^{\rho W_{r}}\left\|e^{\Lambda r} T_{r} f_{1}\right\|_{\infty}^{p-1} d r\right]^{-\frac{1}{p-1}}, \\
& \mathcal{B}_{2}(t)=\left[1-(q-1) \int_{0}^{t} e^{\rho W_{r}}\left\|e^{\Lambda r} T_{r} f_{2}\right\|_{\infty}^{q-1} d r\right]^{-\frac{1}{q-1}} .
\end{aligned}
$$

Then we have

$$
\frac{d \mathcal{B}_{1}(t)}{d t}=e^{\rho W_{t}+(p-1) \Lambda t}\left\|T_{t} f_{1}\right\|_{\infty}^{p-1} \mathcal{B}_{1}^{p}(t), \quad \mathcal{B}_{1}(0)=1
$$

hence

$$
\mathcal{B}_{1}(t)=1+\int_{0}^{t} e^{\rho W_{r}+(p-1) \Lambda r}\left\|T_{r} f_{1}\right\|_{\infty}^{p-1} \mathcal{B}_{1}^{p}(r) d r
$$

and similarly,

$$
\mathcal{B}_{2}(t)=1+\int_{0}^{t} e^{\rho W_{r}+(q-1) \Lambda r}\left\|T_{r} f_{2}\right\|_{\infty}^{q-1} \mathcal{B}_{2}^{q}(r) d r .
$$

Let us choose $v \geq 0$ such that $v(t, x) \leq e^{\Lambda t} T_{t} f_{1}(x) \mathcal{B}_{1}(t)$ for $x \in D$ and $t<\tau_{*}$. Then $e^{\Lambda t} T_{t} f_{1}(x) \leq$ $\mathcal{R}_{1} v(t, x)$ and

$$
\begin{aligned}
\mathcal{R}_{1} v(t, x) & =e^{\Lambda t} T_{t} f_{1}(x)+\int_{0}^{t} e^{\rho W_{r}+\Lambda(t-r)}\left(T_{t-r} v(r, x)\right)^{p} d r \\
& \leq e^{\Lambda t} T_{t} f_{1}(x)+\int_{0}^{t} e^{\rho W_{r}+\Lambda(t-r)} \mathcal{B}_{1}(r)^{p-1} e^{\Lambda r(p-1)}\left\|T_{r} f_{1}\right\|_{\infty}^{p-1} \mathcal{B}_{1}(r) e^{\Lambda r} T_{t-r}\left(T_{r} f_{1}\right)(x) d r \\
& =e^{\Lambda t} T_{t} f_{1}(x)\left[1+\int_{0}^{t} e^{\rho W_{r}}\left\|e^{\Lambda r} T_{r} f_{1}\right\|_{\infty}^{p-1} B_{1}^{p}(r) d r\right] \\
& =e^{\Lambda t} T_{t} f_{1}(x) \mathcal{B}_{1}(t)
\end{aligned}
$$

and similarly,

$$
e^{\Lambda t} T_{t} f_{2}(x) \leq \mathcal{R}_{2} u(t, x) \leq e^{\Lambda t} T_{t} f_{2}(x) \mathcal{B}_{2}(t)
$$

for all $u$ such that $0 \leq u(t, x) \leq e^{\Lambda t} T_{t} f_{2}(x) \mathcal{B}_{2}(t)$. Let us take, for $x \in D$ and $0 \leq t<\tau_{*}$,

$$
u_{1}^{(0)}(t, x)=e^{\Lambda t} T_{t} f_{1}(x), \quad u_{2}^{(0)}(t, x)=e^{\Lambda t} T_{t} f_{2}(x)
$$

and

$$
u_{1}^{(n)}(t, x)=\mathcal{R}_{1} u_{2}^{(n-1)}(t, x), \quad u_{2}^{(n)}(t, x)=\mathcal{R}_{2} u_{1}^{(n-1)}(t, x), \quad n \geq 1 .
$$


We are going to show that the function sequences $\left\{u_{1}^{(n)}\right\},\left\{u_{2}^{(n)}\right\}$ are increasing. As a matter of fact,

$$
u_{1}^{(0)}(t, x) \leq e^{\Lambda t} T_{t} f_{1}(x)+\int_{0}^{t} e^{\rho W_{r}+\Lambda(t-r)}\left(T_{t-r} u_{2}^{(0)}(r, x)\right)^{p} d r=\mathcal{R}_{1} u_{2}^{(0)}(t, x)=u_{1}^{(1)}(t, x) .
$$

Now assume that $u_{1}^{(n)} \geq u_{1}^{(n-1)}$ and $u_{2}^{(n)} \geq u_{2}^{(n-1)}$ for some $n \geq 1$. Then

$$
u_{1}^{(n+1)}=\mathcal{R}_{1} u_{2}^{(n)} \geq \mathcal{R}_{1} u_{2}^{(n-1)}=u_{1}^{(n)},
$$

where we have used the monotonicity of $\mathcal{R}_{1}$ to obtain the above inequality. In the same way is proved the monotonicity of the other sequence. Therefore the limits

$$
v_{1}(t, x)=\lim _{n \rightarrow \infty} u_{1}^{(n)}(t, x), \quad v_{2}(t, x)=\lim _{n \rightarrow \infty} u_{2}^{(n)}(t, x)
$$

exist for $x \in D$ and $0 \leq t<\tau_{*}$. In virtue of the monotone convergence theorem we obtain that

$$
v_{1}(t, x)=\mathcal{R}_{1} v_{2}(t, x), \quad v_{2}(t, x)=\mathcal{R}_{2} v_{1}(t, x), \quad x \in D, \quad 0 \leq t<\tau_{*},
$$

and moreover,

$$
\begin{aligned}
& v_{1}(t, x) \leq \frac{e^{\Lambda t} T_{t} f_{1}(x)}{\left[1-(p-1) \int_{0}^{t} e^{\rho W_{r}}\left\|e^{\Lambda r} T_{r} f_{1}\right\|_{\infty}^{p-1} d r\right]^{\frac{1}{p-1}}}, \\
& v_{2}(t, x) \leq \frac{e^{\Lambda t} T_{t} f_{2}(x)}{\left[1-(q-1) \int_{0}^{t} e^{\rho W_{r}}\left\|e^{\Lambda r} T_{r} f_{2}\right\|_{\infty}^{q-1} d r\right]^{\frac{1}{q-1}}}
\end{aligned}
$$

The assertion follows by choosing the initial values according to (3.2).

Remark Notice that for general bounded, measurable and positive $f_{i}, i=1,2$, the blow-up time of (1.2) is lower-bounded by the random time

$$
\inf \left\{t \geq 0: \int_{0}^{t} e^{\rho W_{r}}\left\|e^{\Lambda r} T_{r} f_{1}\right\|_{\infty}^{p-1} d r \geq(p-1)^{-1} \quad \text { or } \quad \int_{0}^{t} e^{\rho W_{r}}\left\|e^{\Lambda r} T_{r} f_{2}\right\|_{\infty}^{q-1} d r \geq(q-1)^{-1}\right\}
$$

which coincides with $\tau_{*}$ when the initial values satisfy $(3.2)$.

\subsection{An upper bound for $\tau$}

We now set $\varphi_{i}=\psi$ and $V_{i}=\Lambda+\kappa_{i}^{2} / 2$ in (2.3), $i=1,2$, thus obtaining the system

$$
v_{i}(t, \psi)=v_{i}(0, \psi)+\int_{0}^{t} e^{-\kappa_{i} W_{s}}\left(e^{\kappa_{j} W_{s}} v_{j}\right)^{\beta_{j}}(s, \psi) d s, \quad i=1,2,
$$

where $\left(e^{\kappa_{j} W_{s}} v_{j}\right)^{\beta_{i}}(s, \psi)=\int_{D}\left[e^{\kappa_{j} W_{s}} v_{j}(s, x)\right]^{\beta_{i}} \psi(x) d x$ and $\beta_{1}=p, \beta_{2}=q$. By Jensen's inequality,

$$
\left(e^{\kappa_{j} W_{s}} v_{j}\right)^{\beta_{i}}(s, \psi) \geq\left[\int_{D}\left(e^{\kappa_{j} W_{s}} v_{j}(s, x)\right) \psi(x) d x\right]^{\beta_{i}}=e^{\beta_{i} \kappa_{j} W_{s}} v_{j}(s, \psi)^{\beta_{i}},
$$

which gives

$$
\frac{\partial v_{i}(t, \psi)}{\partial t} \geq e^{-\kappa_{i} W_{t}}\left(e^{\beta_{i} \kappa_{j} W_{t}} v_{j}(t, \psi)^{\beta_{i}}\right)=e^{-\kappa_{i} W_{t}+\beta_{i} \kappa_{j} W_{t}} v_{j}(t, \psi)^{\beta_{i}}, \quad i=1,2, \quad j \in\{1,2\} \backslash\{i\} .
$$


In this way, $v_{i}(t, \psi) \geq h_{i}(t), i=1,2$, where

$$
\frac{d h_{1}(t)}{d t}=e^{\rho W_{t}} h_{2}(t)^{p}, \quad \frac{d h_{2}(t)}{d t}=e^{\rho W_{t}} h_{1}(t)^{q}, \quad h_{i}(0)=v_{i}(0, \psi), \quad i=1,2,
$$

and $\rho=p \kappa_{2}-\kappa_{1}=q \kappa_{1}-\kappa_{2}$. We define $E(t)=h_{1}(t)+h_{2}(t), t \geq 0$.

\section{Theorem 2}

1. Assume that $p=q>1$. Then $\tau \leq \tau^{*}$, where

$$
\tau^{*}=\inf \left\{t \geq 0: \int_{0}^{t} e^{\rho W_{s}} d s \geq 2^{p}(p-1)^{-1} E^{1-p}(0)\right\} .
$$

2. Let $p>q>1$, and let $A_{0}=\frac{p-q}{p}\left(\frac{p}{q}\right)^{\frac{q}{p-q}}$ and $\epsilon_{0}=1 \wedge\left(h_{2}(0) / A_{0}^{1 / q}\right)^{p-q} \wedge\left(2^{-q} E(0)^{q} / A_{0}\right)^{(p-q) / p}$. Assume that

$$
2^{-q} \epsilon_{0} E^{q}(0) \geq \epsilon_{0}^{\frac{p}{p-q}} A_{0} .
$$

Then $\tau \leq \tau^{*}$, where

$$
\tau^{*}=\inf \left\{t \geq 0: \int_{0}^{t} e^{\rho W_{s}} d s \geq \frac{1}{(q-1) E^{q-1}(0)\left[2^{-q} \epsilon_{0}-\epsilon_{0}^{p /(p-q)} A_{0} E^{-q}(0)\right]}\right\} .
$$

Remark Notice that (3.5) follows from the condition $\int_{D} f_{i}(x) \psi(x) d x>A_{0}^{1 / q}, i=1,2$.

Proof. Suppose first that $p=q>1$. We get from (3.4) that

$$
\frac{d E(t)}{d t}=e^{\rho W_{t}}\left(h_{1}^{p}(t)+h_{2}^{p}(t)\right) .
$$

By substituting $y \equiv h_{1} / h_{2}$ into the inequality $1+y^{p} \geq 2^{-p}(1+y)^{p}$, which is valid for $y \geq 0$, we obtain

$$
1+\left(\frac{h_{1}(t)}{h_{2}(t)}\right)^{p} \geq 2^{-p}\left(1+\frac{h_{1}(t)}{h_{2}(t)}\right)^{p}
$$

hence $h_{1}^{p}(t)+h_{2}^{p}(t) \geq 2^{-p}\left(h_{1}(t)+h_{2}(t)\right)^{p}$. Plugging this into (3.6) leads to the differential inequality

$$
\frac{d E(t)}{d t} \geq e^{\rho W_{t}} 2^{-p} E^{p}(t) .
$$

Thus, $E(t)$ blows up no later than the solution $I(t)$ of the equation

$$
\frac{d I(t)}{d t}=e^{\rho W_{t}} 2^{-p} I^{p}(t), \quad I(0)=E(0),
$$

whose explosion time is given by

$$
\tau^{*}=\inf \left\{t \geq 0: \int_{0}^{t} e^{\rho W_{s}} d s \geq 2^{p}(p-1)^{-1} E^{1-p}(0)\right\} .
$$

Now we assume that $p>q>1$. We want to lower-bound the solution components of system (3.4). Our approach to achieve this is an adaptation of the method used in [10], where a deterministic system similar to (1.2) was investigated. 
Let us recall Young's inequality (see e.g. [11]). Let $1<b<\infty$ and $\delta>0$, and let $a=b /(b-1)$. Then

$$
x y \leq \frac{\delta^{a} x^{a}}{a}+\frac{\delta^{-b} y^{b}}{b}, \quad x, y \geq 0 .
$$

From Young's inequality (3.7) it follows that for any $\varepsilon>0$,

$$
h_{2}^{p}(t) \geq \varepsilon h_{2}^{q}(t)-\varepsilon^{\frac{p}{p-q}} A_{0} .
$$

Indeed, (3.8) follows from (3.7) by setting $b=p / q, y=h_{2}^{q}(t), x=\epsilon, \delta=(p / q)^{q / p}$, and using that $q<p$. We also have

$$
\epsilon_{0} h_{2}(0)^{q}-\epsilon_{0}^{\frac{p}{p-q}} A_{0} \geq 0 .
$$

In fact, if $\epsilon_{0}=1$ then $1 \leq\left(h_{2}(0) / A_{0}^{1 / q}\right)^{p-q}$, which immediately gives $A_{0} \leq h_{2}^{q}(0)$. If $\epsilon_{0}=$ $\left(h_{2}(0) / A_{0}^{1 / q}\right)^{p-q}$ then $\epsilon_{0} h_{2}^{q}(0)-\epsilon_{0}^{p /(p-q)} A_{0}=\left(h_{2}^{p}(0) / A_{0}^{p / q}\right) \cdot 0=0$, and if $\epsilon_{0}=\left(2^{-q} E^{q}(0) / A_{0}\right)^{(p-q) / p}$ we obtain

$$
\epsilon_{0} h_{2}^{q}(0)-\epsilon_{0}^{p /(p-q)} A_{0}=\frac{2^{-q} E^{q}(0)}{A_{0}}\left[\left(\frac{A_{0}}{2^{-q} E^{q}(0)}\right)^{q / p} h_{2}^{q}(0)-A_{0}\right],
$$

which together with the inequality $\left(A_{0} /\left(2^{-q} E^{q}(0)\right)\right)^{q / p} \geq A_{0} / h_{2}^{q}(0)$, render that the expression in brackets is lower-bounded by zero.

It follows from (3.4) and (3.8) that

$$
\frac{d h_{1}(t)}{d t} \geq e^{\rho W_{t}}\left[\epsilon_{0} h_{2}^{q}(t)-\epsilon_{0}^{\frac{p}{p-q}} A_{0}\right]
$$

and therefore

$$
\frac{d E(t)}{d t} \geq e^{\rho W_{t}}\left[h_{1}^{q}(t)+\epsilon_{0} h_{2}^{q}(t)-\epsilon_{0}^{\frac{p}{p-q}} A_{0}\right] .
$$

Setting $y \equiv \epsilon_{0}^{1 / q} h_{2}(t) / h_{1}(t)$ and using again the inequality $1+y^{q} \geq 2^{-q}(1+y)^{q}$, we see that

$$
h_{1}^{q}(t)+\epsilon_{0} h_{2}^{q}(t)=h_{1}^{q}(t)\left[1+\left(\epsilon_{0}^{1 / q} \frac{h_{2}(t)}{h_{1}(t)}\right)^{q}\right] \geq 2^{-q}\left(h_{1}(t)+\epsilon_{0}^{1 / q} h_{2}(t)\right)^{q} \geq 2^{-q} \epsilon_{0} E^{q}(t)
$$

because $\epsilon_{0} \leq 1$. Plugging this into (3.10) renders

$$
\frac{d E(t)}{d t} \geq e^{\rho W_{t}}\left[2^{-q} \epsilon_{0} E^{q}(t)-\epsilon_{0}^{\frac{p}{p-q}} A_{0}\right] .
$$

Due to assumption (3.5) we get $E(t) \geq E(0)>0$ for any $t \geq 0$. Moreover, (3.11) transforms into

$$
\frac{d E(t)}{E^{q}(t)} \geq e^{\rho W_{t}}\left[2^{-q} \epsilon_{0}-\frac{\epsilon_{0}^{p /(p-q)} A_{0}}{E(0)^{q}}\right] d t
$$

which gives

$$
E^{q-1}(t) \geq \frac{E^{q-1}(0)}{1-(q-1) E^{q-1}(0)\left[2^{-q} \epsilon_{0}-\epsilon_{0}^{p /(p-q)} A_{0} E^{-q}(0)\right] \int_{0}^{t} e^{\rho W_{s}} d s} .
$$

In this way $E(t)$, and therefore $\left(h_{1}(t), h_{2}(t)\right)$, is going to blow-up earlier than the random time $\tau^{*}$ given by

$$
\tau^{*}=\inf \left\{t \geq 0: \int_{0}^{t} e^{\rho W_{s}} d s \geq \frac{1}{(q-1) E^{q-1}(0)\left[2^{-q} \epsilon_{0}-\epsilon_{0}^{p /(p-q)} A_{0} E^{-q}(0)\right]}\right\} .
$$




\section{A more general case}

In this section we consider system (1.2) under the assumptions $p \geq q>1$ and $V_{i}=\Lambda+\kappa_{i}^{2} / 2$, $i=1,2$. Let us again write $\beta_{1}=p, \beta_{2}=q$ and $j \in\{1,2\} \backslash\{i\}$. We have from (2.4)

$$
v_{i}(t, x)=e^{\Lambda t} T_{t} f_{i}(x)+\int_{0}^{t} e^{\left(\beta_{i} \kappa_{j}-\kappa_{i}\right) W_{r}+\Lambda(t-r)} T_{t-r}\left(v_{j}(r, \cdot)^{\beta_{i}}\right)(x) d r, \quad i=1,2 .
$$

Theorem 3 Assume that $p \geq q>1$ and $V_{i}=\Lambda+\kappa_{i}^{2} / 2, i=1,2$, and let $f_{1}=L(1) \psi$ and $f_{2}=L(2) \psi$ for some positive constants $L(1)$ and $L(2)$. Let $\tau_{* *}$ be given by

$$
\begin{aligned}
\tau_{* *}=\inf \{t \geq 0 & : \int_{0}^{t} e^{\left(p \kappa_{2}-\kappa_{1}\right) W_{r}} d r \geq \frac{1}{(p-1) L^{p-1}(1)\|\psi\|_{\infty}^{p-1}} \\
& \text { or } \left.\int_{0}^{t} e^{\left(q \kappa_{1}-\kappa_{2}\right) W_{r}} \geq \frac{1}{(q-1) L^{q-1}(2)\|\psi\|_{\infty}^{q-1}}\right\} .
\end{aligned}
$$

Then $\tau_{* *} \leq \tau$.

Proof. Let us define, for $i=1,2$,

$$
\mathcal{R}_{i} v(t, x)=e^{\Lambda t} T_{t} f_{i}(x)+\int_{0}^{t} e^{\left(\beta_{i} \kappa_{j}-\kappa_{i}\right) W_{r}+\Lambda(t-r)}\left(T_{t-r} v(r, x)\right)^{\beta_{i}} d r,
$$

and

$$
\mathcal{B}_{i}(t)=\left[1-\left(\beta_{i}-1\right) \int_{0}^{t} e^{\left(\beta_{i} \kappa_{j}-\kappa_{i}\right) W_{r}+\Lambda r}\left\|T_{r} f_{i}\right\|_{\infty}^{\beta_{i}-1} d r\right]^{-1 /\left(\beta_{i}-1\right)} .
$$

Proceeding in the same way as in the proof of Theorem 1, we get that

$$
v_{1}(t, x)=\mathcal{R}_{1} v_{2}(t, x), \quad v_{2}(t, x)=\mathcal{R}_{2} v_{1}(t, x)
$$

as long as $t \leq \tau_{* *}$ and $x \in D$. Moreover,

$$
\begin{aligned}
v_{i}(t, x) & \leq \frac{e^{\Lambda t} T_{t} f_{i}(x)}{\left[1-\left(\beta_{i}-1\right) \int_{0}^{t} e^{\left(\beta_{i} \kappa_{j}-\kappa_{i}\right) W_{r}}\left\|e^{\Lambda r} T_{r} f_{i}\right\|_{\infty}^{\beta_{i}-1} d r\right]^{\frac{1}{\beta_{i}-1}}} \\
& =\frac{L(i) \psi(x)}{\left[1-\left(\beta_{i}-1\right) L(i)^{\beta_{i}-1}\|\psi\|_{\infty}^{\beta_{i}-1} \int_{0}^{t} e^{\left(\beta_{i} \kappa_{j}-\kappa_{i}\right) W_{r}} d r\right]^{\frac{1}{\beta_{i}-1}}}
\end{aligned}
$$

by our choice of $f_{1}$ and $f_{2}$.

Corollary 4 Let the random time $\tau^{\prime}$ be defined by

$$
\begin{aligned}
\tau^{\prime}=\inf \left\{t \geq 0: \int_{0}^{t} \max \right. & \left\{e^{\left(p \kappa_{2}-\kappa_{1}\right) W_{r}}, e^{\left(q \kappa_{1}-\kappa_{2}\right) W_{r}}\right\} d r \\
& \left.\geq \min \left\{\frac{1}{(p-1) L^{p-1}(1)\|\psi\|_{\infty}^{p-1}}, \frac{1}{(q-1) L^{q-1}(2)\|\psi\|_{\infty}^{q-1}}\right\}\right\} .
\end{aligned}
$$

Then $\tau^{\prime} \leq \tau_{* *}$ 
In order to obtain an upper bound for $\tau$ when $p \geq q>1$ and $V_{i}=\Lambda+\kappa_{i}^{2} / 2, i=1,2$, we first notice that a sub-solution for Eq. (3.3) is given by the solution of

$$
\frac{d h_{1}(t)}{d t}=e^{\left(p \kappa_{2}-\kappa_{1}\right) W_{t}} h_{2}(t)^{p}, \quad \frac{d h_{2}(t)}{d t}=e^{\left(q \kappa_{1}-\kappa_{2}\right) W_{t}} h_{1}(t)^{q}, \quad h_{i}(0)=v_{i}(0, \psi), \quad i=1,2 .
$$

Working with system (4.1) as we did with (3.4) in the proof of Theorem 2, we get that

$$
\frac{d E(t)}{d t} \geq \min \left\{e^{\left(p \kappa_{2}-\kappa_{1}\right) W_{t}}, e^{\left(q \kappa_{1}-\kappa_{2}\right) W_{t}}\right\}\left[2^{-q} \epsilon_{0} E^{q}(t)-\epsilon_{0}^{\frac{p}{p-q}} A_{0}\right],
$$

and in case of $p=q$,

$$
\frac{d E(t)}{d t} \geq \min \left\{e^{\left(p \kappa_{2}-\kappa_{1}\right) W_{t}}, e^{\left(p \kappa_{1}-\kappa_{2}\right) W_{t}}\right\} 2^{-p} E^{p}(t)
$$

which can be handled using the same method as in the proof of Theorem 2.1. In this way we obtain the following result for the explosion time of system (1.2).

Theorem 5 Let $p \geq q>1$.

1. If $p=q$ then $\tau \leq \tau^{* *}$, where

$$
\tau^{* *}=\inf \left\{t \geq 0: \int_{0}^{t} \min \left\{e^{\left(p \kappa_{2}-\kappa_{1}\right) W_{r}}, e^{\left(p \kappa_{1}-\kappa_{2}\right) W_{r}}\right\} d s \geq 2^{p}(p-1)^{-1} E^{1-p}(0)\right\} .
$$

2. If $p>q$ and (3.5) holds true, then $\tau \leq \tau^{* *}$ where

$$
\begin{aligned}
\tau^{* *}=\inf \{t \geq 0: & \int_{0}^{t} \min \left\{e^{\left(p \kappa_{2}-\kappa_{1}\right) W_{r}}, e^{\left(q \kappa_{1}-\kappa_{2}\right) W_{r}}\right\} d r \\
& \left.\geq \frac{1}{(q-1) E^{q-1}(0)\left[2^{-q} \epsilon_{0}-\epsilon_{0}^{p /(p-q)} A_{0} E^{-q}(0)\right]}\right\} .
\end{aligned}
$$

Remark Setting $t \geq 0$ and

$$
M:= \begin{cases}\left((q-1) E^{q-1}(0)\left[2^{-q} \epsilon_{0}-\epsilon_{0}^{p /(p-q)} A_{0} E^{-q}(0)\right]\right)^{-1}, & \text { if } p>q, \\ 2^{p}(p-1)^{-1} E^{1-p}(0), & \text { if } p=q,\end{cases}
$$

we get

$$
\begin{aligned}
\mathbb{P}\left\{\tau^{* *} \geq t\right\} & =\mathbb{P}\left\{\int_{0}^{t} \min \left\{e^{\left(p \kappa_{2}-\kappa_{1}\right) W_{r}}, e^{\left(q \kappa_{1}-\kappa_{2}\right) W_{r}}\right\} d r \leq M\right\} \\
& =\mathbb{P}\left\{e^{-\int_{0}^{t} \min \left\{e^{\left(p \kappa_{2}-\kappa_{1}\right) W_{r}}, e^{\left(q \kappa_{1}-\kappa_{2}\right) W_{r}}\right\} d r} \geq e^{-M}\right\} \\
& \leq e^{M} \mathbb{E}\left[e^{-\int_{0}^{t} \min \left\{e^{\left.\left(p \kappa_{2}-\kappa_{1}\right) W_{r}, e^{\left(q \kappa_{1}-\kappa_{2}\right) W_{r}}\right\} d r}\right]},\right.
\end{aligned}
$$

where we used Chebyshev's inequality. A similar estimate for the distribution function of $\tau_{* *}$, but involving the functional $\int_{0}^{t} \max \left\{e^{\left(p \kappa_{2}-\kappa_{1}\right) W_{r}}, e^{\left(q \kappa_{1}-\kappa_{2}\right) W_{r}}\right\} d r$, can be obtained using that $\tau^{\prime} \leq \tau_{* *}$. The Laplace transforms of the random functionals $\int_{0}^{\theta} \max \left\{e^{a W_{r}}, e^{b W_{r}}\right\} d r, \int_{0}^{\theta} \min \left\{e^{a W_{r}}, e^{b W_{r}}\right\} d r$ where $\theta$ is an independent exponentially distributed time and $a>0, b>0$ are constants, are given in Theorem 7 below. 


\section{The Laplace transform of a Brownian motion functional}

Let $a, b \in(0, \infty)$ be given constants, and let $F: \mathbb{R} \rightarrow(0, \infty)$ be the locally bounded function defined by

$$
F(z)=e^{a z} \mathbf{1}_{[0, \infty)}(z)+e^{b z} \mathbf{1}_{(-\infty, 0)}(z) .
$$

Hence,

$$
F(z)= \begin{cases}\min \left\{e^{a z}, e^{b z}\right\}, & \text { if } a \leq b, \\ \max \left\{e^{a z}, e^{b z}\right\}, & \text { if } a \geq b .\end{cases}
$$

We want to investigate the functional of Brownian motion $\mathcal{A}_{t}^{F}$ defined by

$$
\mathcal{A}_{t}^{F}=\int_{0}^{t} F\left(W_{s}\right) d s=\int_{0}^{t}\left(e^{a W_{s}} \mathbf{1}_{[0, \infty)}\left(W_{s}\right)+e^{b W_{s}} \mathbf{1}_{(-\infty, 0)}\left(W_{s}\right)\right) d s .
$$

Let $\theta_{k}$ be an exponentially distributed random variable of parameter $k$, independent of $\left\{W_{s}\right\}$, and let $g_{\theta_{k}}(\omega)$ be the last zero of $W .(\omega)$ before $\theta_{k}(\omega)$. Our first aim is to calculate the Laplace transform of $\mathcal{A}_{\theta_{k}}^{F}$, which follows from a simple adaptation of the approaches in [5] and [14]. In fact, as in [5] we can write

$$
\mathbb{E}\left[\exp \left\{-\mathcal{A}_{\theta_{k}}^{F}\right\}\right]=\mathbb{E}\left[\exp \left\{-\mathcal{A}_{g_{\theta_{k}}}^{F}\right\}\right] \mathbb{E}\left[\exp -\left\{\mathcal{A}_{\theta_{k}}^{F}-\mathcal{A}_{g_{\theta_{k}}}^{F}\right\}\right]
$$

and substituting $\lambda F$ instead of $F$ in the above equality, where $\lambda \geq 0$, we get an expression for the Laplace transform of $\mathcal{A}_{\theta_{k}}^{F}$ in terms of the two expectations in the RHS of (5.2). Let $\left\{\ell_{t}\right\}$ be the local time at 0 of $\left\{W_{s}\right\}$, and

$$
\tau_{s}:=\inf \left\{t \geq 0: \ell_{t}>s\right\}
$$

the inverse local time. Using formula (2.4) in [5] we obtain that

$$
\mathbb{E}\left[\exp \left\{-\mathcal{A}_{g_{\theta_{k}}}^{F}\right\}\right]=k \int_{0}^{\infty} d l \mathbb{E}\left[\exp -\left\{\frac{k^{2}}{2} \tau_{l}+\mathcal{A}_{\tau_{l}}^{F}\right\}\right]
$$

From Corollary 3.4 and Theorem 3.1 in [5] we know that for general $F$,

$$
\mathbb{E}\left[\exp -\left\{\frac{k^{2}}{2} \tau_{l}+\mathcal{A}_{\tau_{l}}^{F}\right\}\right]=\exp \left\{\frac{l}{2}\left(\Phi^{F_{+}}\right)_{t}^{\prime}(k, 0)+\frac{l}{2}\left(\Phi^{F_{-}}\right)_{t}^{\prime}(k, 0)\right\},
$$

where $\Phi^{F}(k, t)=: u(k, t)$ is the unique bounded solution of the Sturm-Liouville equation

$$
\frac{1}{2} U^{\prime \prime}=\left(\frac{k^{2}}{2}+F\right) U, \quad U(0)=1,
$$

and $F_{+}:=\left.F\right|_{\mathbb{R}_{+}}, F_{-}(z):=F(-z), z \geq 0$. In our case

$$
F_{+}(z)=e^{a z} \quad \text { and } \quad F_{-}(z)=e^{-b z}, \quad z \geq 0
$$

The remaining expectation in the RHS of (5.2) can be written as

$$
\mathbb{E}\left[\exp \left\{-\mathcal{A}_{\theta_{k}}^{F}-\mathcal{A}_{g_{\theta_{k}}}^{F}\right\}\right]=\frac{k}{2} \int_{0}^{\infty} d t\left[\Phi^{F_{+}}(k, t)+\Phi^{F_{-}}(k, t)\right]
$$


see [5], Corollary 3.2. We are going to calculate the functions $\Phi^{\frac{\lambda^{2}}{2}} F_{+}(k, t)$ and $\Phi^{\frac{\lambda^{2}}{2}} F_{-}(k, t)$ in our special setting. The Sturm-Liouville equation corresponding to $\frac{\lambda^{2}}{2} F_{+}, \lambda \geq 0$, is given by

$$
\frac{1}{2} U^{\prime \prime}(a, k, t)=\left(\frac{k^{2}}{2}+\frac{\lambda^{2}}{2} e^{a t}\right) U(a, k, t), \quad U(a, k, 0)=1 .
$$

When $a=2$ we can use Yor's solution of the previous equation, according to which

$$
U(2, k, t)=\frac{K_{k}\left(\lambda e^{t}\right)}{K_{k}(\lambda)}=: \Phi^{\frac{\lambda^{2}}{2}} F_{+}(k, t)
$$

where $K_{\nu}$ is the usual modified Bessel function with index $\nu$. When $a \neq 2$ we solve (5.6) by means of a change of variables. We set $t=2 s / a$ and for simplicity denote $U(a, k, t)$ by $v(k, t)$. Then we have,

$$
\frac{1}{2} v_{t t}^{\prime \prime}\left(k, \frac{2 s}{a}\right)=\left(\frac{k^{2}}{2}+\frac{\lambda^{2}}{2} e^{2 s}\right) v\left(k, \frac{2 s}{a}\right), \quad v(k, 0)=1,
$$

and

$$
v_{t}^{\prime}=v_{s}^{\prime} \frac{d s}{d t}=\frac{a}{2} v_{s}^{\prime}, \quad v_{t t}^{\prime \prime}=\left(\frac{a}{2}\right)^{2} v_{s s}^{\prime \prime}
$$

Therefore,

$$
\frac{1}{2} \frac{a^{2}}{4} v_{s s}^{\prime \prime}\left(k, \frac{2 s}{a}\right)=\left(\frac{k^{2}}{2}+\frac{\lambda^{2}}{2} e^{2 s}\right) v\left(k, \frac{2 s}{a}\right)
$$

or

$$
\frac{1}{2} v_{s s}^{\prime \prime}\left(k, \frac{2 s}{a}\right)=\left(\frac{2 k^{2}}{a^{2}}+\frac{2 \lambda^{2}}{a^{2}} e^{2 s}\right) v\left(k, \frac{2 s}{a}\right) .
$$

Putting $w(k, s)=v(k, 2 s / a)$, we get $w^{\prime}(k, s)=(2 / a) v^{\prime}(k, 2 s / a)$ and $\left(w^{\prime \prime}\right)^{2}(k, s)=\frac{4}{a^{2}} v^{\prime \prime}(k, 2 s / a)$. Hence, $w$ satisfies

$$
\frac{1}{2} \frac{a^{2}}{4} w^{\prime \prime}(k, s)=\left(\frac{2 k^{2}}{a^{2}}+\frac{2 \lambda^{2}}{a^{2}} e^{2 s}\right) w(k, s),
$$

and

$$
\frac{1}{2} w^{\prime \prime}(k, s)=\left(\frac{8 k^{2}}{a^{4}}+\frac{8 \lambda^{2}}{a^{4}} e^{2 s}\right) w(k, s),
$$

which is the equation (5.6) with $a=2$ and new constants $k$ and $\lambda$. Thus, by (5.7), $w$ has the form

$$
w(k, t)=\frac{K_{k^{\prime}}\left(\lambda^{\prime} e^{t}\right)}{K_{k^{\prime}}\left(\lambda^{\prime}\right)} \quad \text { with } \quad k^{\prime}=\frac{4 k}{a^{2}}, \lambda^{\prime}=\frac{4 \lambda}{a^{2}}
$$

and therefore

$$
\Phi^{\frac{\lambda^{2}}{2} F_{+}}(k, t)=v(k, t)=w(k, a t / 2)=\frac{K_{4 k / a^{2}}\left(4 \lambda e^{a t / 2} / a^{2}\right)}{K_{4 k / a^{2}}\left(4 \lambda / a^{2}\right)} .
$$

To calculate $\Phi^{\frac{\lambda^{2}}{2}} F_{-}$we proceed in the same fashion, but now using the fact that the corresponding Sturm-Liouville equation (for $a=2$ ) has as solution

$$
U(2, k, t)=\Phi^{F_{-}}(k, t)=\frac{I_{k}\left(\lambda e^{-t}\right)}{I_{k}(\lambda)}, \quad t \geq 0
$$

where $I_{k}$ is the other modified Bessel function. Hence,

$$
\Phi^{\frac{\lambda^{2}}{2} F_{-}}(k, t)=\frac{I_{4 k / b^{2}}\left(4 \lambda e^{b t / 2} / b^{2}\right)}{I_{4 k / b^{2}}\left(4 \lambda / b^{2}\right)}, \quad t \geq 0
$$


We now continue with the development of (5.4). We recall from [14] (pag. 131) that

$$
\lambda K_{k+1}(\lambda)=-\lambda K_{k}^{\prime}(\lambda)+k K_{k}(\lambda) \quad \text { and } \quad \lambda I_{k-1}(\lambda)=\lambda I_{k}^{\prime}(\lambda)+k I_{k}(\lambda)
$$

where the derivatives are with respect to $\lambda$. The derivatives of $\Phi^{\frac{\lambda^{2}}{2}} F_{+}(k, t)$ and $\Phi^{\frac{\lambda^{2}}{2}} F_{-}(k, t)$ at the point $t=0$ are given by

$$
\begin{aligned}
\left(\Phi^{\frac{\lambda^{2}}{2}} F_{+}\right)_{t}^{\prime}(k, 0) & =\frac{K_{4 k / a^{2}}^{\prime}\left(4 \lambda / a^{2}\right)(a / 2)\left(4 \lambda / a^{2}\right)}{K_{4 k / a^{2}}\left(4 \lambda / a^{2}\right)} \\
& =\frac{2 \lambda}{a}\left(\frac{4 k /\left(a^{2}\right)}{4 \lambda /\left(a^{2}\right)}-\frac{K_{\frac{4 k}{a^{2}}+1}\left(4 \lambda / a^{2}\right)}{K_{4 k / a^{2}}\left(4 \lambda / a^{2}\right)}\right) \\
& =\frac{a}{2}\left(\frac{4 k}{a^{2}}-\frac{\left(4 \lambda / a^{2}\right) K_{4 k / a^{2}+1}\left(4 \lambda / a^{2}\right)}{K_{4 k / a^{2}}\left(4 \lambda / a^{2}\right)}\right),
\end{aligned}
$$

where in the last equality we used (5.8). In a similar way,

$$
\left(\Phi^{\frac{\lambda^{2}}{2}} F_{-}\right)_{t}^{\prime}(k, 0)=\frac{I_{4 k / b^{2}}^{\prime}\left(4 \lambda / b^{2}\right)(b / 2)\left(4 \lambda / b^{2}\right)}{I_{4 k / b^{2}}\left(4 \lambda / b^{2}\right)}=\frac{b}{2}\left(\frac{\left(4 \lambda / b^{2}\right) I_{4 k / b^{2}-1}\left(4 \lambda / b^{2}\right)}{I_{4 k / b^{2}}\left(4 \lambda / b^{2}\right)}-\frac{4 k}{b^{2}}\right) .
$$

Substituting the two expressions above into (5.4) we obtain the following.

Lemma 6 For all $a, b \in(0, \infty)$,

$$
\mathbb{E}\left[\exp -\left\{\frac{k^{2}}{2} \tau_{l}+\frac{\lambda^{2}}{2} \mathcal{A}_{\tau_{l}}^{F}\right\}\right]=\exp \left\{-\frac{l}{2}(R(a, \lambda, k)+S(b, \lambda, k))\right\}
$$

where

$$
R(a, \lambda, k)=\frac{a}{2}\left(\frac{\frac{4 \lambda}{a^{2}} K_{\frac{4 k}{a^{2}}+1}\left(\frac{4 \lambda}{a^{2}}\right)}{K_{\frac{4 k}{a^{2}}}\left(\frac{4 \lambda}{a^{2}}\right)}-\frac{4 k}{a^{2}}\right) \quad \text { and } S(b, \lambda, k)=\frac{b}{2}\left(\frac{4 k}{b^{2}}-\frac{\frac{4 \lambda}{b^{2}} I_{\frac{4 k}{b^{2}}-1}\left(\frac{4 \lambda}{b^{2}}\right)}{I_{\frac{4 k}{b^{2}}}\left(\frac{4 \lambda}{b^{2}}\right)}\right) .
$$

Putting together (5.2), (5.3), (5.5) and Lemma 6 we get the following formulas.

Theorem 7 Let $a, b \in(0, \infty)$. The Laplace transforms of $\mathcal{A}_{g_{\theta_{k}}}^{F}$ and $\mathcal{A}_{\theta_{k}}^{F}$ are given by

$$
\mathbb{E}\left[e^{-\frac{\lambda^{2}}{2} \mathcal{A}_{g_{\theta_{k}}}^{F}}\right]=k \int_{0}^{\infty} \exp \left\{-\frac{l}{2}(R(a, \lambda, k)+S(b, \lambda, k))\right\} d l=\frac{2 k}{R(a, \lambda, k)+S(b, \lambda, k)},
$$

and

$$
\mathbb{E}\left[e^{-\frac{\lambda^{2}}{2} \mathcal{A}_{\theta_{k}}^{F}}\right]=\frac{k^{2}}{R(a, \lambda, k)+S(b, \lambda, k)} \int_{0}^{\infty}\left[\frac{K_{\frac{4 k}{a^{2}}}\left(\frac{4 \lambda}{a^{2}} e^{\frac{a x}{2}}\right)}{K_{\frac{4 k}{a^{2}}}\left(\frac{4 \lambda}{a^{2}}\right)}+\frac{I_{\frac{4 k}{b^{2}}}\left(\frac{4 \lambda}{b^{2}} e^{\frac{b x}{2}}\right)}{I_{\frac{4 k}{b^{2}}}\left(\frac{4 \lambda}{b^{2}}\right)}\right] d x
$$

where $R(a, \lambda, k)$ and $S(b, \lambda, k)$ are given by (5.9).

Corollary 8 Let $\theta_{k}$ be an exponentially distributed random variable of parameter $k>0$, independent of $\left\{W_{t}\right\}$, and let $P:=\min \left\{p \kappa_{2}-\kappa_{1}, q \kappa_{1}-\kappa 2\right\}$ and $Q:=\max \left\{p \kappa_{2}-\kappa_{1}, q \kappa_{1}-\kappa 2\right\}$ be strictly positive. Then,

$$
\mathbb{P}\left\{\tau \geq \theta_{k}\right\} \leq \frac{k^{2} e^{M}}{R(P, \sqrt{2}, k)+S(Q, \sqrt{2}, k)} \int_{0}^{\infty}\left[\frac{K_{\frac{4 k}{P^{2}}}\left(\frac{4 \lambda}{P^{2}} e^{\frac{P x}{2}}\right)}{K_{\frac{4 k}{P^{2}}}\left(\frac{4 \lambda}{P^{2}}\right)}+\frac{I_{\frac{4 k}{Q^{2}}}\left(\frac{4 \lambda}{Q^{2}} e^{\frac{Q x}{2}}\right)}{I_{\frac{4 k}{Q^{2}}}\left(\frac{4 \lambda}{Q^{2}}\right)}\right] d x,
$$

where $M$ is given by (4.2), and $R$ and $S$ are given by (5.9). 
Proof. From the definition of $\tau^{* *}$ (see Theorem 5) we know that for any $s \geq 0$,

$$
\mathbb{P}\{\tau \geq s\} \leq \mathbb{P}\left\{\tau^{* *} \geq s\right\}=\mathbb{P}\left\{\mathcal{A}_{s}^{F} \leq M\right\}
$$

where $\mathcal{A}_{s}^{F}$ is given by (5.1) with $a=P$ and $b=Q$. Using Chebyshev's inequality as before we get $\mathbb{P}\left\{\mathcal{A}_{s}^{F} \leq M\right\} \leq e^{M} \mathbb{E}\left[\exp \left\{-\mathcal{A}_{s}^{F}\right\}\right]$. The result follows from (5.11), putting $\lambda=\sqrt{2}$ and $s=\theta_{k}$.

Acknowledgement. The authors are grateful to an anonymous referee for valuable comments, and for letting them know about reference [8]. This research was partially supported by the CNRS-CONACyT project grant "Blow-up of parabolic stochastic partial differential equations." The first named author acknowledges the hospitality of CIMAT, Guanajuato, and the second and third named authors acknowledge the hospitality of Institut Elie Cartan, Nancy, where part of this work was done. The authors acknowledge the hospitality of Bernoulli Center, EPF Lausanne, during the thematic semester on stochastic analysis.

\section{References}

[1] Bailleul, I. Une preuve simple d'un résultat de Dufresne. Séminaire de Probabilités XLI, 203-213 (2008).

[2] Dozzi, M. and López-Mimbela, J.A. Finite-time blowup and existence of global positive solutions of a semi-linear SPDE. Stochastic Processes and their Applications, 120(6), 767776.

[3] D. Dufresne, The distribution of a perpetuity, with applications to risk theory and pension funding. Scand. Actuarial J. (1990), 39-79.

[4] Fujita, H. On some nonexistence and nonuniqueness theorems for nonlinear parabolic equations, in: Nonlinear Functional Analysis, Providence, R.I., 1970, Proc. Sympos. Pure Math. 18 (1) (1968) 105-113.

[5] Jeanblanc, M.; Pitman, J.; Yor, M. The Feynman-Kac formula and decomposition of Brownian paths. Mat. Apl. Comput. 16 (1997), no. 1, 27-52.

[6] Geman, H. and Yor, M. Bessel Processes, Asian Options, and Perpetuities. Mathematical Finance 33, No. 4 (1993), 349-375.

[7] Matsumoto, H. and Yor, M. Exponential functionals of Brownian motion, I: Probability laws at a fixed time. Probability Surveys 2 (2005), 312-347.

[8] Pintoux, C. and Privault, N. A direct solution to the Fokker-Planck equation for exponential Brownian functionals. Analysis and Applications 8 (2010), 287-304.

[9] Salminen P. and Yor, M. Properties of perpetual integral functionals of Brownian motion with drift, Annales de l'Institute Henri Poincare - Probabilites et Statistiques, Vol. 41, p. $335-347,2005$. 
[10] Galaktionov, V.A., Kurdyumov, S.P. and Samarskii-, A.A. A parabolic system of quasilinear equations. II. Differential Equations 21 (1985), no. 9, 1049-1062.

[11] Mitrinovic, D.S. Analytic inequalities. In cooperation with P. M. Vasic. Die Grundlehren der mathematischen Wissenschaften, Band 165. Springer-Verlag, New York-Berlin 1970.

[12] Tehranchi, M. The distribution of exponential Lévy functionals. Preprint (2009).

[13] Yor, M. On Some Exponential Functionals of Brownian Motion. Adv. Appl. Prob. 24 (1992), 509-531.

[14] Yor, M. Exponential Functionals of Brownian Motion and Related Processes. Springer Finance, 2001. 